\title{
A Study of Potential Pharmacokinetic and Pharmacodynamic Interactions between Dextromethorphan/Quinidine and Memantine in Healthy Volunteers
}

\author{
Laura E. Pope, ${ }^{1}$ Kerri A. Schoedel, ${ }^{2}$ Cynthia Bartlett ${ }^{2}$ and Edward M. Sellers ${ }^{3}$
}

1 Avanir Pharmaceuticals, Inc., Aliso Viejo, CA, USA

2 INC Research - Toronto, Toronto, Canada (formerly, at the time of the study, Ventana Clinical Research Corporation)

3 DL Global Partners Inc. (Ventana Clinical Research Corporation at the time of the study), Toronto, Canada

\section{Abstract}

Background and Objective: Dextromethorphan/quinidine (DMQ) is the first agent indicated for the treatment of pseudobulbar affect. Dextromethorphan, the active ingredient, is a low-affinity, uncompetitive $N$-methyl-D-aspartate (NMDA) receptor antagonist. This study evaluated the potential for a drugdrug interaction (DDI) of DMQ with memantine, which is also an NMDA receptor antagonist.

Methods: This open-label, randomized, parallel-group study enrolled healthy adults who were randomized into one of two treatment groups. Group 1 subjects were administered memantine at a starting dose of $5 \mathrm{mg}$ once daily, which was titrated over a 3-week period to a dose of $10 \mathrm{mg}$ twice daily (every 12 hours) and continued for another 11 days to attain steady state; DMQ $30 \mathrm{mg}$ (dextromethorphan $30 \mathrm{mg}$ /quinidine $30 \mathrm{mg}$ ) every 12 hours was then added for a further 8 days. Group 2 subjects received DMQ $30 \mathrm{mg}$ every 12 hours for 8 days to attain steady state; memantine was then added, titrated on the same schedule as in group 1, and continued at $10 \mathrm{mg}$ every 12 hours for an additional 11 days. Pharmacokinetic blood sampling was performed to assess the primary endpoints of the $90 \%$ confidence intervals (CIs) for the geometric mean ratios of the areas under the plasma concentration-time curves (AUCs) for memantine, dextromethorphan, dextrorphan - the dextromethorphan metabolite - and quinidine during concomitant therapy versus monotherapy. Safety/tolerability and pharmacodynamic variables were also assessed.

Results: A total of 52 subjects were randomized. In both group $1(n=23)$ and group $2(n=29)$, the $90 \%$ CIs for the ratios of the AUCs during concomitant therapy versus monotherapy were within the predefined range to indicate similarity (0.8-1.25) for memantine, dextromethorphan and dextrorphan, indicating no pharmacokinetic DDI. The $90 \%$ CI for the AUC ratio for 
quinidine was slightly above the predefined range; however, the mean AUC increased by only $25 \%$. In both groups, incidence of adverse events was similar, and pharmacodynamic variables were either similar or slightly improved with DMQ added to memantine and memantine added to DMQ, compared to monotherapy with either agent.

Conclusion: Minimal pharmacokinetic and pharmacodynamic interactions were observed between memantine and DMQ, suggesting they can be coadministered without dose adjustment.

\section{Background}

Pseudobulbar affect (PBA) is a neurological disorder of emotional affect, characterized by involuntary, sudden and uncontrollable outbursts of laughing and/or crying, which are often exaggerated or incongruous to the stimulus or social context. ${ }^{[1-3]}$ This syndrome occurs secondary to neurological disease or brain injury, including Alzheimer's disease or dementia, multiple sclerosis, Parkinson's disease, amyotrophic lateral sclerosis, stroke and traumatic brain injury, among other conditions. ${ }^{[2]} \mathrm{PBA}$ episodes often cause severe embarrassment and social dysfunction, and can lead to social isolation, reduced quality of life and impaired function for patients, independent of the burdens of the underlying, primary disorder. ${ }^{[1,2,4-9]}$

Dextromethorphan/quinidine (DMQ) [Nuedexta, Avanir Pharmaceuticals, Inc., Aliso Viejo, CA, USA; 2010] is the first agent indicated for the treatment of PBA, based on evidence of its safety and efficacy in randomized, controlled clinical trials. ${ }^{[10-12]}$ The US FDA-approved dosage of DMQ is dextromethorphan $20 \mathrm{mg}$ and quinidine $10 \mathrm{mg}$ taken every 12 hours. ${ }^{[13]}$ Dextromethorphan, the pharmacologically active ingredient of DMQ, is a low-affinity, uncompetitive $N$-methylD-aspartate (NMDA) receptor antagonist, ${ }^{[14]}$ a serotonin reuptake inhibitor ${ }^{[15]}$ and a sigma-1 receptor agonist. ${ }^{[16]}$ Dextromethorphan is metabolized by the polymorphic cytochrome P450 (CYP) 2D6, and undergoes rapid hepatic conversion to its metabolite dextrorphan, such that plasma concentrations of dextromethorphan are too low in most patients to achieve therapeutic effect, even at high doses. ${ }^{[17-19]}$ For this reason, dextromethorphan is combined with quinidine, a CYP2D6 inhibitor, which has been shown to increase the systemic availability of dextromethorphan by blocking its first-pass hepatic metabolism. ${ }^{[19]}$ Dextromethorphan levels when coadministered with quinidine (dextromethorphan $30 \mathrm{mg}+$ quinidine $30 \mathrm{mg}$ ) after repeated dosing have been reported to be 30 - to 40 -fold higher than levels of dextromethorphan administered alone. ${ }^{[17]}$

Because patients with PBA have underlying neurological conditions that may be treated with a range of medications, and DMQ is a relatively new agent to treat PBA, it is important to evaluate the potential for drug-drug interactions. Memantine is a voltage-dependent, moderate-affinity, uncompetitive NMDA receptor antagonist, which is indicated for the treatment of patients with moderate to severe Alzheimer's disease. ${ }^{[20]}$ Although there are limited data available on the prevalence of PBA in this population, it has been estimated that up to $39 \%$ of patients with Alzheimer's disease also experience PBA. ${ }^{[2,21]}$ Therefore, physicians may consider treating patients with Alzheimer's disease with memantine and DMQ concomitantly. Since both agents are NMDA receptor antagonists, however, their coadministration could, theoretically, lead to an additive effect at NMDA receptors and increased rates or severity of adverse events (AEs). ${ }^{[20]}$ In addition, quinidine may theoretically inhibit memantine clearance, since both agents undergo renal tubular transport and urinary excretion. ${ }^{[22-24]}$ Therefore, a study was conducted to assess the pharmacokinetic and pharmacodynamic drugdrug interactions resulting from coadministration of DMQ and memantine, and the safety and tolerability of this combination. Of note, the dosage of DMQ used in this study was dextromethor- 
phan $30 \mathrm{mg}$ /quinidine $30 \mathrm{mg}$ every 12 hours since it was the DMQ dosage being investigated at the time of the study, but is higher than the dosage ultimately approved for treatment of PBA (see above). ${ }^{[13]}$

\section{Methods}

\section{Study Design}

This was an open-label, randomized, parallelgroup clinical study in healthy adult subjects, conducted at a single site, INC Research - Toronto (Ventana Clinical Research Corporation, Toronto, Canada, at the time of the study). The study was composed of a screening visit and a treatment period, and subjects were randomized to two treatment groups.
In group 1, subjects were administered memantine at a starting dose of $5 \mathrm{mg}$ once daily, which was titrated over a 3 -week period to a dose of $10 \mathrm{mg}$ twice daily (every 12 hours) [figure 1]. Upon reaching the memantine $10 \mathrm{mg}$ every 12 hours dose (day 22), group 1 subjects continued this treatment for 11 days to attain steady-state drug plasma levels, based on reported clinical data. ${ }^{[25]}$ DMQ $30 \mathrm{mg}$ (dextromethorphan $30 \mathrm{mg} /$ quinidine $30 \mathrm{mg}$ ) every 12 hours was then added (starting day 33) to the ongoing memantine treatment for the next 8 days. In group 2, subjects received DMQ $30 \mathrm{mg}$ every 12 hours for 8 days to attain steady state (figure 1), and memantine was then added (starting day 9) to the ongoing DMQ therapy, with memantine administered at a starting dose of $5 \mathrm{mg}$ once daily and titrated up to $10 \mathrm{mg}$ every 12 hours as in group 1; once the target

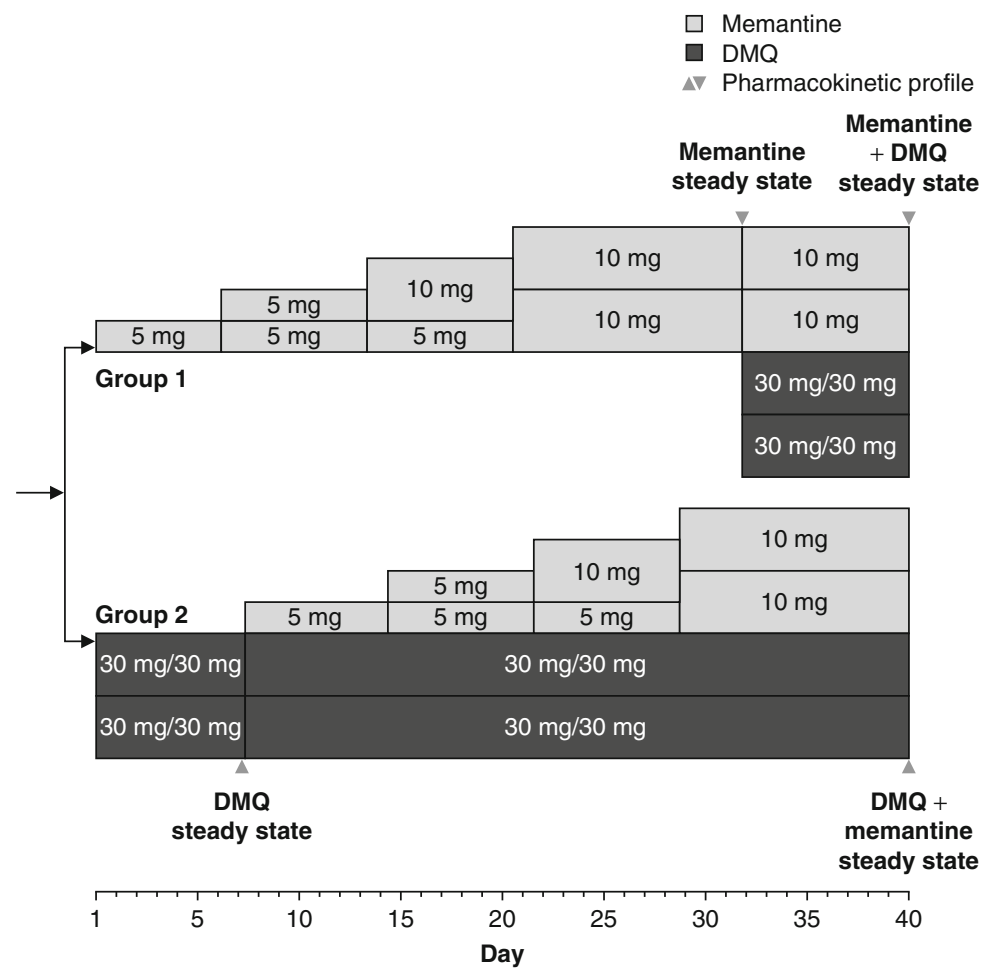

Fig. 1. Study design. In group 1 (top), subjects underwent 3-week titration of memantine to the target dose of $10 \mathrm{mg}$ every 12 hours, followed by continuation of memantine $10 \mathrm{mg}$ every 12 hours for 11 days to achieve steady state, and then the addition of DMQ 30 mg every 12 hours for another 8 days. In group 2 (bottom), subjects took DMQ 30 mg every 12 hours for 8 days to achieve steady state. Memantine was then added to DMQ $30 \mathrm{mg}$ on the same titration schedule as in group 1 until the target dose (memantine 10 mg every 12 hours) was reached, and then the concomitant therapy was continued for another 11 days. On the final day of monotherapy and of concomitant therapy, blood sampling was performed in each group for steady-state pharmacokinetic analysis (vertical arrows). DMQ = dextromethorphan $30 \mathrm{mg} / \mathrm{quinidine} 30 \mathrm{mg}$. 
memantine dose was reached (day 30), the DMQ and memantine regimens were continued for an additional 11 days. Pre-dose plasma samples were obtained at several time points for both memantine and DMQ to confirm whether steadystate drug levels had been attained by the designated time point (day 11 for memantine, day 8 for DMQ).

This study was conducted in accordance with the ethical principles stated in the Declaration of Helsinki, 1964, amended in Edinburgh in 2000, and Good Clinical Practice. Prior to initiation of the study, the protocol, consent form, amendments to the protocol and advertisements for subject recruitment were reviewed and approved by the Institutional Review Board of the participating study centre. Before participating in this study, all subjects were required to sign informed, written consent forms that encompassed all procedures and assessments to be performed during the study, including genotyping.

\section{Study Objectives}

The primary objectives of this study were to assess in healthy adults: the effect of DMQ on the steady-state pharmacokinetics of memantine; the effect of memantine on the steady-state pharmacokinetics of DMQ; and the safety and tolerability of coadministration of DMQ and memantine. The secondary objectives of this study were to assess the effects of the addition of DMQ on selected pharmacodynamic measures associated with memantine, and the effect of the addition of memantine on selected pharmacodynamic measures associated with DMQ.

\section{Study Population}

\section{Eligibility Criteria}

Eligible subjects were healthy men and women aged 19-55 years of age (inclusive) who were nonsmokers (for at least 3 months prior to the screening visit); had a body weight of at least $60 \mathrm{~kg}$ for men and at least $52 \mathrm{~kg}$ for women, and a body mass index (BMI) of $19-30 \mathrm{~kg} / \mathrm{m}^{2}$, inclusive; were free from any clinically significant abnormality of medical history; were able to abstain from alcohol (ethanol) from at least 2 weeks prior to the start of the treatment period and until final discharge, and had negative breath alcohol tests at screening, and at each outpatient visit to the clinical research unit. Women of reproductive potential who were not abstinent from sex with men were entered into the study if they were using and would continue to use adequate contraception, and had a negative serum pregnancy test at the screening visit and throughout the study.

\section{Exclusion Criteria}

Subjects were excluded if they had a history or present condition of major organ or systemic disease or psychiatric illness; substance abuse or dependence; renal impairment (creatinine clearance $\leq 50 \mathrm{~mL} / \mathrm{min}$ ); myasthenia gravis, conjunctivitis or urinary tract infections; severe gastrointestinal narrowing or hepatic or renal impairment that could interfere with drug absorption, distribution, metabolism or elimination; a PR interval $\geq 190 \mathrm{msec}$ at the screening visit or $\geq 200 \mathrm{msec}$ prior to the first dose of study medication; corrected QT (QTc) prolongation (were taking medications or had a condition known to prolong QTc, or whose QTc interval was $>450 \mathrm{msec}$ for men or $>470 \mathrm{msec}$ for women at the screening visit); had used any drugs or substances known to be strong inhibitors or inducers of CYP enzymes within 30 days of the first study dose; had used any prescription (except oral contraceptives or sex hormone replacement therapy) or non-prescription drugs or natural health products (except vitamins/minerals and paracetamol [acetaminophen] up to $2 \mathrm{~g}$ /day) within 14 days of the first dose and for the duration of the study; use of monoamine oxidase inhibitors, thioridazine, pimozide, serotonin-precursors and other serotonergic drugs, antipsychotics, barbiturates, anti-epileptics, dantrolene or baclofen within 21 days of the first study dose.

\section{Other Restrictions and Prohibitions}

Subjects were required to fast for approximately 8 hours prior to dosing on days requiring post-dose pharmacokinetic sampling, and 2 hours prior to dosing to 1 hour post dosing on all other study days. Water was not permitted from 2 hours pre-dose until 1 hour post-dose, except for the $125 \mathrm{~mL}$ of non-carbonated water that was taken 
during each study drug administration. At all other times water was permitted ad libitum. Subjects also agreed to abstain for at least 2 weeks prior to the first dose administration until final discharge from foods and/or beverages that could interfere with laboratory values or drug metabolism. Strenuous exercise (defined as any activity that resulted in a heart rate that was $70 \%$ or more of the maximum resting heart rate) was not permitted 48 hours prior to admission (treatment period) and throughout the treatment period until final discharge.

\section{Study Outcomes}

\section{Pharmacokinetic Variables}

The primary pharmacokinetic outcome variables of this study were the $90 \%$ confidence intervals (CIs) of the true geometric mean ratios for:

- Memantine area under the plasma concentration-time curve from 0 to 12 hours $\left(\mathrm{AUC}_{12}\right)$ on day 40 (memantine with DMQ) and day 32 (memantine alone);

- DMQ (dextromethorphan, dextrorphan and quinidine) $\mathrm{AUC}_{12}$ on day 40 (DMQ with memantine) and day 8 (DMQ alone).

Maximum steady-state concentration $\left(\mathrm{C}_{\text {max,ss }}\right)$ and minimum steady-state concentration $\left(\mathrm{C}_{\mathrm{min}, \mathrm{ss}}\right)$, time to reach maximum concentration $\left(t_{\max }\right)$ and the apparent elimination half-life $\left(t_{1 / 2}\right)$ were also examined for memantine and DMQ. To control for genotype status, genotyping analysis of $C Y P 2 D 6$ was conducted for each subject.

\section{Blood Samples Collection and Analysis}

Blood samples were collected at designated times via an optional indwelling catheter or by direct venipuncture for assessment of drug levels. Blood samples for pharmacokinetic assessments were obtained for the morning dose at 1 hour predose and 1, 2, 3, 4, 5, 6, 8 and 12 hours post-dose for DMQ, and at 1 hour pre-dose and 2, 4, 6, 8, 10 and 12 hours post-dose for memantine. In group 1 pharmacokinetic assessments were conducted for memantine on day 32, and for memantine and DMQ on day 40; in group 2, pharmacokinetic assessments were conducted for DMQ on day 8 , and for DMQ and memantine on day 40. Ad- ditionally, to monitor progression to steady state, blood sampling was performed within 5 minutes prior to the first morning dose administration on the following days: group 1 days 2, 5, 8, 11, 14, 17, $20,23,26$ and 29-31 to assess memantine levels and days 37-39 to assess DMQ levels, and group 2 days $2,5-7,10,13,16,19,22,25$ and 28 to assess DMQ levels and days 10, 13, 16, 19, 22, 25, 28 and 37-39 to assess memantine levels.

In order to conduct dextromethorphan, dextrorphan and quinidine assays, a $6 \mathrm{~mL}$ blood sample was collected in a heparinized tube; for memantine assays, a $5 \mathrm{~mL}$ blood sample was collected in an ethylenediaminetetraacetic acidcontaining tube. All blood samples were cooled in an ice bath prior to processing and separated by centrifugation at approximately $2500 \mathrm{rpm}$ for 15 minutes at $4{ }^{\circ} \mathrm{C}$. Following centrifugation, two plasma aliquots per sample were placed in clearly labelled polypropylene containers and stored in a freezer at $-20^{\circ} \mathrm{C}$ or below until removed for shipping; sample aliquots were shipped on dry ice in two separate shipments, with the second set of aliquots shipped after notification of receipt of the first. Approximately $202 \mathrm{~mL}$ of blood were collected during the course of the study for pharmacokinetic sampling in group 1 (26 blood draws for memantine [130 mL] and 12 blood draws for DMQ [72 $\mathrm{mL}$ ]) and $259 \mathrm{~mL}$ in group 2 (17 blood draws for memantine [85 $\mathrm{mL}$ ] and 29 blood draws for DMQ [174 mL]).

MDS Pharma Services, Lincoln, NE, USA, and Sittingbourne, UK, performed all analyses of the plasma samples. The three bioanalytical methods used for these analyses were all conducted under Principles of Good Laboratory Practice described in the Code of Federal Regulations title 21, part 58 and the Guidance for Industry Bioanalytical Method Validation (Center for Drug Evaluation and Research, May 2001). The plasma samples for dextromethorphan and dextrorphan were analysed by using a liquid chromatography-tandem mass spectrometry (LC-MS/MS) assay. In this assay, internal standards (d3-dextromethorphan hydrochloride and d3-dextrorphan hydrobromide) were spiked into the plasma, and the mixture was treated for enzymatic hydrolysis and then extracted into an organic solvent. The 
organic phase was transferred to a clean tube, evaporated to dryness and reconstituted for injection on an LC-MS/MS (Applied Biosystems/ MDS Sciex API 4000). For dextromethorphan, the linearity range was $0.2 \mathrm{ng} / \mathrm{mL}$ to $200 \mathrm{ng} / \mathrm{mL}$ with a lower limit of quantitation (LLQ) of $0.2 \mathrm{ng} / \mathrm{mL}$; for dextrorphan these parameters were $2.5 \mathrm{ng} / \mathrm{mL}$ to $2500 \mathrm{ng} / \mathrm{mL}$ with a LLQ of $2.5 \mathrm{ng} / \mathrm{mL}$.

Quinidine was assayed using a validated highperformance liquid chromatography (HPLC) assay. Quinidine and the internal standard (quinine) were isolated from plasma by protein precipitation with acetonitrile. Samples were diluted with water before injection onto the HPLC. The linearity range was $2-250 \mathrm{ng} / \mathrm{mL}$, and the LLQ was $2 \mathrm{ng} / \mathrm{mL}$. Memantine was assayed using a validated LC-MS/MS assay. An aliquot of the study plasma sample containing added internal standard (1-amantylamine hydrochloride) was prepared using a solid-phase extraction procedure. The extracted samples were analysed using an HPLC equipped with a MDS Sciex API 4000 mass spectrometer. Quantification was by peak area ratio. The linearity range was $0.1-30 \mathrm{ng} / \mathrm{mL}$, and the LLQ was $0.1 \mathrm{ng} / \mathrm{mL}$.

For all assays, a single set of calibration standards placed near the beginning of each batch defined a standard curve from which six replicates of quality control samples at three concentrations were determined. There were no significant interfering peaks. Selectivity was demonstrated against possible contaminants and metabolites. Interday and intraday variability fell below $15 \%$ (coefficient of variation and relative error).

For CYP2D6 genotyping analysis, a $10 \mathrm{~mL}$ whole blood sample was collected from each subject at any time on day -1 of the first treatment period. Each sample was collected into a lavender top polypropylene EDTA Vacutainer ${ }^{\circledR}$ or equivalent and gently inverted ten times to ensure proper mixing of the anticoagulant with the blood sample. Samples were processed by Genaissance, Morrisville, NC, USA for $C Y P 2 D 6 * 3$, $* 4, * 5, * 6, * 7$ and *8 alleles by multiplex polymerase chain reaction. ${ }^{[26]}$ The analyses of the CYP $2 D 6 *{ }^{*} 5,{ }^{*} 2 \mathrm{XN},{ }^{*} 10$ and $* 17$ alleles were each done in separate assays. Based on the results, predicted metabolizer phenotypes of extensive metabolizer (EM), poor metabolizer (PM), intermediate metabolizer (IM) or ultra-rapid metabolizer (UM) were assigned for each sample. Each sample was to be stored at Genaissance for no longer than 2 years and then destroyed, having been used only to determine CYP2D6 genotype status.

\section{Safety and Tolerability Variables}

Safety and tolerability measures included physical examination, vital signs, 12-lead electrocardiogram (ECG), laboratory tests and monitoring of AEs, including serious AEs (SAEs). Urine drug screens were conducted at baseline and after the last evening drug administration on days $8,15,22$ and 31 in group 1, and on days 7, 9, 16, 23 and 28 in group 2. AEs were reported and recorded throughout the study from the time informed consent was obtained until discharge from the study, including at follow-up (for SAEs following the last dose of study drug), and were coded using the Medical Dictionary for Regulatory Activities (MedDRA).

\section{Pharmacodynamic Variables}

The secondary study outcomes were pharmacodynamic measures selected based on the sideeffect profiles of the two drugs and included the following: (1) The Choice Reaction Time (CRT), a test of psychomotor function measuring speed and accuracy with which subjects use a keypad to input sequences of numbers that are displayed on a corresponding computer screen image; the test measures Recognition Reaction Time (RRT), Motor Reaction Time (MRT) and Total Reaction Time (RRT+MRT). ${ }^{[27]}$ (2) The Divided Attention Test (DAT), a manual tracking and visual target detection task comprised of an image of an airplane and a randomly curving road. Subjects are asked to use a joystick to keep the airplane over the road while simultaneously responding to random visual targets. ${ }^{[28]}$ (3) The Postural Stability Test, which uses a platform to measure variables pertaining to body sway and postural stability. Subjects are asked to stand on the platform for over 1 minute with their eyes open and then with their eyes closed. Summary variables are the area of a $95 \%$ confidence ellipse (to track time-related 
sway in balance), the length of the path of the centre-of-pressure coordinates and the total test time completed. ${ }^{[29]}$ (4) Visual analogue scales (VAS) for nausea and dizziness consisting of a horizontal line ranging from 0 to 100 with descriptive anchor endpoints of 'not at all' and 'extremely'. Subjects were asked to indicate on the scale their responses to the statements "I feel dizzy", and "I feel nauseous". (5) The Beck Depression Inventory, 2nd Edition (BDI-II), a 21-item self-report scale measuring presence and degree of depressive symptoms over the past 2 weeks. Responses to each item correspond to a scale from 0 (neutral) to 3 (maximum severity), with a total possible score of $63 .{ }^{[30]}$ (6) The Beck Anxiety Inventory (BAI), a 21-item self-report scale measuring common symptoms of anxiety. The subject rates each item (ranging from 0 to 3 ) based on how much he/she has been bothered by each symptom over the past week (total score $0-63$ ). ${ }^{31]}$

(7) The Leeds Sleep Evaluation Questionnaire (LSEQ), ${ }^{[32]}$ a 10 -item self-rating scale measuring perceived changes in sleep, ease of wakening and alertness.

The pharmacodynamic measures were assessed during peak drug levels (after the last morning dose) on days $1,8,15,22,32,33,36$ and 40 in group 1 and on days 1, 8, 9, 15, 22, 29, 36 and 40 in group 2. The CRT, DAT, Postural Stability and VAS scales were assessed at 2, 4 and 6 hours; the LSEQ at 2 hours; and the BAI and BDI-II at 4 hours after dosing. A training session was conducted on day -1 in both groups, and all subjects underwent a pharmacodynamic refresher session before dosing on the morning on which pharmacodynamic measures were collected.

\section{Statistical Methods}

The sample size was planned to include 40 healthy volunteers ( 20 per treatment group) with the intention of approximately 12 subjects completing in each treatment group. For a test of equivalence, a sample size of 12 would have had a power of $80 \%$, given that the limit of equivalence was $89 \%$ of the standard deviation of the difference between the two conditions. The safety population included all randomized subjects who took at least one dose of any study drug. The evaluable population included all subjects in the safety population who completed the study, with no major protocol violations that would exclude them from analysis, and for whom calculation of the primary pharmacokinetic measures was possible. Demographic and other baseline characteristics of the study population were characterized using the safety population and with standard descriptive statistics.

For group 1 subjects, to address the primary hypothesis of similarity of memantine $\mathrm{AUC}_{12}$ with and without DMQ, the individual natural log-transformed memantine $\mathrm{AUC}_{12}$ values were assessed in an analysis of variance (ANOVA) model with factors for treatment (memantine with DMQ or memantine alone) and subject. A 90\% $\mathrm{CI}$ for the natural $\log$ scale mean $\mathrm{AUC}_{12}$ difference (memantine after 8 days of DMQ - memantine alone) was calculated. The limits of the 90\% CI were exponentiated to obtain a $90 \%$ CI for the memantine $\mathrm{AUC}_{12}$ true geometric mean ratio (memantine after 8 days of DMQ/ memantine alone). A CI of within 0.8 and 1.25 would support the primary hypothesis. Tests of significance were not appropriate because small, consistent systemic exposure differences can be statistically significant $(\mathrm{p}<0.05)$ but not clinically relevant. A similar procedure was used for $\mathrm{C}_{\max , \mathrm{ss}}$ and $\mathrm{C}_{\text {min,ss. }}$. Summary statistics (minimum, maximum, median or harmonic mean) were computed for the memantine $t_{\max }$ and apparent $t_{1 / 2}$ values for each treatment (memantine with DMQ or memantine alone). In addition, a plot of the memantine pre-dose concentrations was examined to confirm whether steady state had been attained by 31 days of memantine treatment.

Similar procedures were conducted in group 2 subjects to address the primary hypothesis for this group of similarity of DMQ (separately for dextromethorphan, dextrorphan and quinidine) $\mathrm{AUC}_{12}$ with and without memantine. The individual log-transformed DMQ $\mathrm{AUC}_{12}$ values were evaluated in an ANOVA model with factors for treatment (DMQ with memantine and DMQ alone) and subject, including only subjects in the DMQ + memantine treatment group. A 90\% CI for the natural $\log$ scale mean $\mathrm{AUC}_{12}$ difference 
(DMQ after 31 days of memantine - DMQ alone) was calculated. The same limits of the $90 \% \mathrm{CI}$ as in group 1 were used for the DMQ AUC 12 true geometric mean ratio (DMQ after 31 days of memantine or DMQ alone) to assess whether the primary group 2 hypothesis was supported. Similar procedures as in group 1 were used in group 2 to report and analyse $\mathrm{C}_{\max , \mathrm{ss}}, \mathrm{C}_{\mathrm{min}, \mathrm{ss}}$, $t_{\max }$ and apparent $t_{1 / 2}$, and to plot pre-dose DMQ concentrations (dextromethorphan, dextrorphan and quinidine) to confirm whether steady state was attained by 8 days of DMQ administration.

With regard to safety and tolerability variables, AEs were reported and recorded throughout the treatment and follow-up periods and were coded using MedDRA Version 9.1; only treatment-emergent AEs were included (i.e. those that began or worsened after the start of treatment). Laboratory tests, vital signs and ECG results were summarized using descriptive statistics. Change from baseline was summarized only for assess- ments that had been made at similar time points on different days.

The evaluable population was used for analysis of pharmacodynamic outcomes. For each treatment group, pharmacodynamic values were evaluated in an ANOVA model with factors for treatment (DMQ + memantine or one drug alone) and subject. A $90 \%$ CI was calculated for the difference. No tests of significance were done.

\section{Results}

\section{Subject Disposition and Baseline Characteristics}

A total of 52 subjects were randomized to group $1(n=23)$ or group $2(n=29)$ [figure 2]. With the exception of one subject in group 2 who withdrew consent before the study start, all randomized subjects received at least one dose of study treatment and were included in the safety population $(n=51)$. The evaluable population

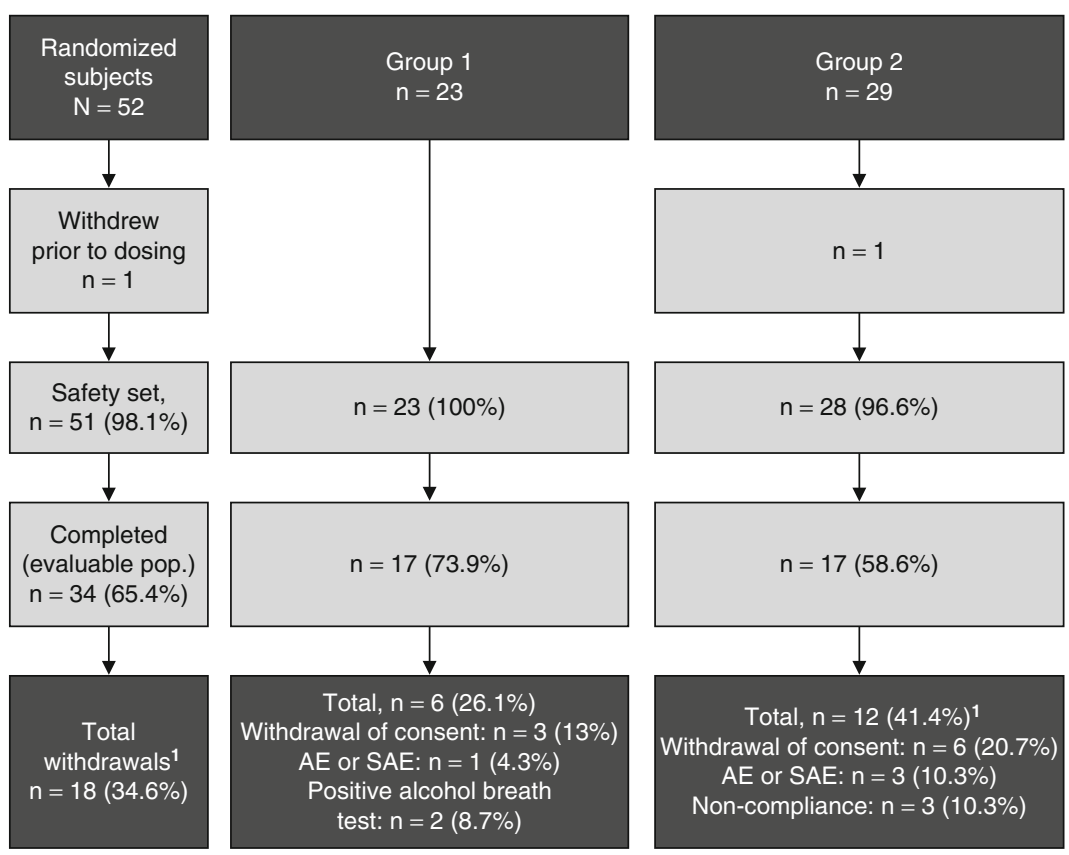

1 One subject randomized to group 2 withdrew consent before study start.

Fig. 2. Subject disposition. $\mathbf{A E}=$ adverse event; pop. = population; $\mathbf{S A E}=$ serious $\mathrm{AE}$. 
Table I. Study subjects' baseline characteristics

\begin{tabular}{|c|c|c|c|}
\hline Characteristic & $\begin{array}{l}\text { Group } 1 \\
(\mathrm{n}=23)\end{array}$ & $\begin{array}{l}\text { Group } 2 \\
(\mathrm{n}=29)\end{array}$ & $\begin{array}{l}\text { Total } \\
(\mathrm{N}=52)\end{array}$ \\
\hline Age, y \{mean (SD) [range]\} & $\begin{array}{l}33.9(9.7) \\
{[20-54]}\end{array}$ & $\begin{array}{l}37.9(9.5) \\
{[19-55]}\end{array}$ & $\begin{array}{l}36.1(9.7) \\
{[19-55]}\end{array}$ \\
\hline \multicolumn{4}{|l|}{ Sex, n (\%) } \\
\hline Male & $15(65.2)$ & $24(82.8)$ & $39(75.0)$ \\
\hline Female & $8(34.8)$ & $5(17.2)$ & $13(25.0)$ \\
\hline \multicolumn{4}{|l|}{ Race, n (\%) } \\
\hline White & $7(30.4)$ & $12(41.4)$ & $19(36.5)$ \\
\hline Asian & $4(17.4)$ & $6(20.7)$ & $10(19.2)$ \\
\hline Black or of African descent & $5(21.7)$ & $4(13.8)$ & $9(17.3)$ \\
\hline Hispanic or Latino & $5(21.7)$ & $6(20.7)$ & $11(21.2)$ \\
\hline Other & $2(8.7)$ & $1(3.4)$ & $3(5.8)$ \\
\hline Weight, kg [mean (SD)] & $76.9(9.5)$ & $76.1(9.1)$ & $76.5(9.2)$ \\
\hline BMI, $\mathrm{kg} / \mathrm{m}^{2}$ [mean (SD)] & $25.9(2.6)$ & $25.7(2.3)$ & $25.8(2.4)$ \\
\hline \multicolumn{4}{|c|}{ CYP2D6 predicted metabolizer phenotype, $\mathrm{n}(\%)^{\mathrm{a}}$} \\
\hline Extensive & $19(82.6)$ & $27(96.4)$ & $46(90.2)$ \\
\hline Intermediate & $1(4.3)$ & $0(0.0)$ & $1(2.0)$ \\
\hline Ultra-rapid & $3(13.0)$ & $1(3.6)$ & $4(7.8)$ \\
\hline \multicolumn{4}{|c|}{$\begin{array}{l}\mathrm{a}=28 \text { in group } 2 \text { as one subject randomized to group } 2 \text { withdrew consent before the start of the study; predicted metabolizer phenotype at } \\
\text { baseline, based on each patient's CYP2D6 genotype. }\end{array}$} \\
\hline \multicolumn{4}{|c|}{$\mathbf{B M I}=$ body mass index; $\mathbf{C Y P}=$ cytochrome $\mathbf{P} 450 ; \mathbf{S D}=$ standard deviation. } \\
\hline
\end{tabular}

was comprised of 34 (65.4\%) subjects, 17 in each group. A total of 18 (34.6\%) randomized subjects did not complete the trial, six (26.1\%) in group 1 and $12(41.4 \%)$ in group 2, including four $(7.7 \%)$ subjects who withdrew from the trial because of an $\mathrm{AE}$ (one [4.3\%] in group 1, and three [10.3\%] in group 2).

The randomized population was mostly male (75\%) with a mean age of 36.1 years and of varying racial descent (table I). Age, body weight and BMI did not differ markedly between the treatment groups, although there were slight differences in sex and race distribution (table I). Most subjects ( $\mathrm{n}=46$ [90.2\%]) were genotyped as CYP2D6 EMs, with four subjects (7.8\%) genotyped as a UM and one subject $(2.0 \%)$ as an IM (table I).

\section{Pharmacokinetic Variables}

\section{Pharmacokinetic Parameters and Effects of Concomitant Therapy}

Examination of pre-dose concentration data indicated that steady state was generally achieved for all dose groups and treatments (table II). In group 1, the mean, steady-state, pre-dose plasma concentration of memantine given as monotherapy (day $32[84.0 \mathrm{ng} / \mathrm{mL}]$ ) was comparable to the pre-dose memantine concentration (day 40 [77.7 ng/mL]) after 8 days of coadministration with DMQ (table II). The $90 \%$ CIs of the ratios of geometric means for $\mathrm{AUC}_{12}$ fell within the interval of $0.8-1.25$, indicating plasma concentrations of memantine given alone compared with memantine in combination with DMQ are similar (figure 3). Mean steady-state $\mathrm{AUC}_{12}$ values of memantine were also similar in group 1 on day 32 (memantine monotherapy), group 1 on day 40 (after the addition of DMQ) and group 2 on day 40 (after the addition of memantine to DMQ monotherapy) [table II]. In addition, the $90 \%$ CIs of the ratios of geometric means for $\mathrm{C}_{\max , \mathrm{ss}}(0.936)$ and $\mathrm{C}_{\mathrm{min}, \mathrm{ss}}$ (0.935) were also within the limits of $0.8-1.25$.

In group 2, the mean pre-dose plasma concentration of dextromethorphan on day 8 of DMQ monotherapy was $65.4 \mathrm{ng} / \mathrm{mL}$, compared with $76.7 \mathrm{ng} / \mathrm{mL}$ on day 40 with memantine coadministration (table II). The $90 \%$ CIs for the ratios of geometric means for $\mathrm{AUC}_{12}$ (figure 3) 
and $\mathrm{C}_{\max , \text { ss }}$ for day 40 fell entirely within the limits of $0.8-1.25$, indicating that dextromethorphan plasma concentrations were similar before and after the addition of memantine. Mean steady-state $\mathrm{AUC}_{12}$ dextromethorphan values were also consistent during DMQ monotherapy, and when DMQ was added to memantine in group 1 , and memantine was added to DMQ in group 2 (table II).

For dextrorphan, the $90 \%$ CIs for the ratio of geometric means of $\mathrm{AUC}_{12}$ (figure 3 ), $\mathrm{C}_{\text {max,ss }}$ and $\mathrm{C}_{\text {min,ss }}$ for day 40 compared with day 8 all fell within the $0.8-1.25$ limits, indicating no differential

Table II. Steady-state pharmacokinetic assessments, evaluable population ${ }^{a}$

\begin{tabular}{|c|c|c|c|c|}
\hline \multirow[t]{2}{*}{ Pharmacokinetic parameter } & \multicolumn{2}{|l|}{ Group 1} & \multicolumn{2}{|l|}{ Group 2} \\
\hline & $\begin{array}{l}\text { Monotherapy: } \\
\text { memantine, day } 32 \\
(n=17)\end{array}$ & $\begin{array}{l}\text { Combination: memantine }+ \\
\text { DMQ, day } 40 \\
(n=17)\end{array}$ & $\begin{array}{l}\text { Monotherapy: } \\
\text { DMQ, day } 8 \\
(n=17)\end{array}$ & $\begin{array}{l}\text { Combination: } \mathrm{DMQ}+ \\
\text { memantine, day } 40 \\
(\mathrm{n}=17)\end{array}$ \\
\hline \multicolumn{5}{|l|}{$\mathrm{C}_{\min , \mathrm{ss}}[$ arithmetic mean (SD)] } \\
\hline Memantine (ng/mL) & $84.0(26.8)$ & 77.7 (30.3) & & $79.3(24.1)$ \\
\hline Dextromethorphan (ng/mL) & & $59.8(28.0)$ & $65.4(18.4)$ & $76.7(22.0)$ \\
\hline Dextrorphan (ng/mL) & & $63.1(37.3)$ & $66.7(20.7)$ & $77.4(28.6)$ \\
\hline Quinidine (ng/mL) & & $50.0(40.0)$ & $50.0(40.0)$ & $70.0(40.0)$ \\
\hline \multicolumn{5}{|l|}{$\mathrm{AUC}_{12}$ [arithmetic mean (SD)] } \\
\hline Memantine $(\mathrm{ng} / \mathrm{mL})$ & $1160.5(360.2)$ & 1111.6 (391.3) & & $1219.5(282.6)$ \\
\hline Dextromethorphan (ng/mL) & & $981.8(374.7)$ & $969.3(248.7)$ & $1060.9(250.4)$ \\
\hline Dextrorphan (ng/mL) & & $778.0(437.1)$ & $753.3(213.8)$ & $818.3(215.6)$ \\
\hline Quinidine (ng/mL) & & $1200.0(500.0)$ & $1000.0(400.0)$ & $1300.0(500.0)$ \\
\hline \multicolumn{5}{|l|}{$\mathrm{C}_{\text {max,ss }}$ [arithmetic mean (SD)] } \\
\hline Memantine (ng/mL) & $106.4(32.8)$ & $102.7(35.6)$ & & $106.4(23.8)$ \\
\hline Dextromethorphan (ng/mL) & & $94.9(33.9)$ & $98.6(24.0)$ & $108.8(24.2)$ \\
\hline Dextrorphan $(\mathrm{ng} / \mathrm{mL})$ & & $71.0(36.3)$ & $72.7(21.6)$ & $81.6(27.5)$ \\
\hline Quinidine (ng/mL) & & $200.0(50.0)$ & $200.0(40.0)$ & $200.0(100.0)$ \\
\hline \multicolumn{5}{|l|}{$\mathrm{C}_{\min , \mathrm{ss}}$ [arithmetic mean (SD)] } \\
\hline Memantine $(\mathrm{ng} / \mathrm{mL})$ & $81.6(26.0)$ & $76.6(30.0)$ & & $77.1(22.7)$ \\
\hline Dextromethorphan (ng/mL) & & $56.9(25.4)$ & $62.8(18.5)$ & $72.9(19.4)$ \\
\hline Dextrorphan (ng/mL) & & $51.2(33.6)$ & $57.1(16.9)$ & $60.8(15.3)$ \\
\hline Quinidine (ng/mL) & & $40.0(30.0)$ & $30.0(30.0)$ & $50.0(40.0)$ \\
\hline \multicolumn{5}{|l|}{$t_{\max }(h)$ [median (range)] } \\
\hline Memantine & $4.00(0.0,12.0)$ & $4.00(2.0,6.0)$ & & $5.00(3.0,9.0)$ \\
\hline Dextromethorphan & & $3.00(3.0,7.0)$ & $3.00(1.0,6.0)$ & $3.00(2.0,5.0)$ \\
\hline Dextrorphan & & $3.00(0.0,13.0)$ & $3.00(0.0,12.0)$ & $1.00(0.0,12.0)$ \\
\hline Quinidine & & $2.00(2.0,3.0)$ & $2.00(1.0,3.0)$ & $1.00(1.0,2.0)$ \\
\hline \multicolumn{5}{|c|}{$\mathrm{t}_{1 / 2}(\mathrm{~h})\{$ arithmetic mean (SD) [median]\} } \\
\hline Memantine & $\begin{array}{l}(n=14) \\
39.7(18.7)[33.7]\end{array}$ & $\begin{array}{l}(n=16) \\
33.4(15.4)[30.7]\end{array}$ & & $\begin{array}{l}(n=16) \\
24.1(11.7)[19.3]\end{array}$ \\
\hline Dextromethorphan & & $19.1(10.2)[16.8]$ & $21.0(12.2)[16.7]$ & $19.4(6.3)[18.5]$ \\
\hline Dextrorphan & & $\begin{array}{l}(n=2) \\
25.5(27.0)[25.5]\end{array}$ & $\begin{array}{l}(n=4) \\
55.0(17.4)[55.6]\end{array}$ & $\begin{array}{l}(n=3) \\
86.6(27.0)[77.6]\end{array}$ \\
\hline Quinidine & & $7.3(2.0)[7.6]$ & $7.0(2.1)[6.9]$ & $6.7(1.8)[6.3]$ \\
\hline
\end{tabular}

a Sample timings relative to administration of drug differ from group 1 to group 2; hence, direct comparison is not appropriate.

$\mathbf{A U C}_{\mathbf{1 2}}=$ area under the plasma concentration-time curve from 0 to 12 hours; $\mathbf{C}_{\text {max,ss }}=$ maximum steady-state concentration; $\mathbf{C}_{\text {min,ss }}=$ minimum steady-state concentration; $\mathbf{D M Q}=$ dextromethorphan $30 \mathrm{mg} /$ quinidine $30 \mathrm{mg}$ every 12 hours; $\mathbf{S D}=$ standard deviation; $\mathbf{t}_{1 / 2}=$ elimination half-life; $\mathbf{t}_{\max }=$ time to reach maximum concentration. 


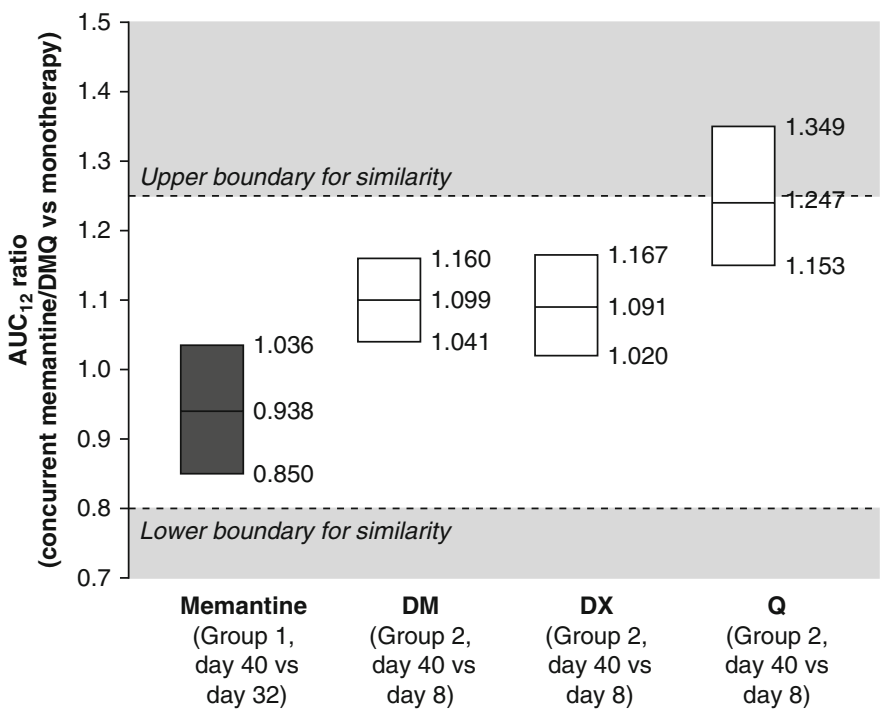

Fig. 3. Ratio of geometric mean area under the plasma concentration-time curve $\left(A \cup C_{12}\right)$ values at steady state of memantine, dextromethorphan (DM), dextrorphan (DX) and quinidine (Q) for memantine and dextromethorphan/quinidine (DMQ) given concomitantly versus memantine or DMQ monotherapy. The geometric mean ratio is represented by the midline of each box; the $90 \%$ confidence intervals (Cls) for a given ratio are indicated by values at the top and bottom of each box. The Cls of the geometric mean ratios were required to be within the predefined interval of $0.8-1.25$ (dotted boundary lines) for treatments to be considered pharmacokinetically similar; $n=17$.

effect of memantine addition on dextrorphan plasma concentrations, compared with DMQ monotherapy. In addition, mean steady-state $\mathrm{AUC}_{12}$ values of dextrorphan were similar during DMQ monotherapy, when memantine was added to DMQ and DMQ was added to memantine monotherapy (table II). For quinidine, the $90 \%$ CIs for the geometric means of $\mathrm{AUC}_{12}$ (figure 3), $\mathrm{C}_{\text {max,ss }}$ and $\mathrm{C}_{\text {min,ss }}$ for day 40 compared with day 8 were above the upper limit of $0.8-1.25$, indicating that the addition of memantine to DMQ increased quinidine plasma concentrations. However, due to the minimal quinidine plasma concentrations produced by the low $30 \mathrm{mg}$ dose of quinidine in the DMQ formulation, the absolute mean differences in quinidine plasma concentrations were small (e.g. mean $\mathrm{C}_{\text {max,ss }}$ was higher by approximately $0.03 \mu \mathrm{g} / \mathrm{mL}$ on day 40 than on day 8 ) [table II].

\section{Safety and Tolerability}

Overall, AEs occurred in 18 (78.3\%) subjects in group 1 and in $26(92.9 \%)$ subjects in group 2. No deaths or SAEs occurred during the study.
Memantine monotherapy was associated with a lower incidence of subjects reporting AEs ( 9 of 20 [45.0\%]) than with DMQ monotherapy (25 of 28 [89.3\%]) [table III]. The incidence and severity of AEs were also greater during DMQ monotherapy than during combination therapy in either order of administration. In group 1, the addition of DMQ to memantine monotherapy was associated with a greater number of subjects reporting at least one AE (14 of 19 subjects [73.7\%]) than during the previous memantine monotherapy at the $10 \mathrm{mg}$ every 12 hours target dose (9 of 20 subjects [45.0\%]) [table III]. However, in group 2, fewer subjects reported at least one AE following the addition of memantine to DMQ (14 of 20 subjects $[70.0 \%]$ ), compared with DMQ monotherapy (25 of 28 subjects [89.3\%]) [table III]. The AE pattern in group 2 gave no indication of a substantial drug interaction; indeed, the total incidence and severity of AEs were actually lower with combination therapy than with DMQ monotherapy.

The most common AEs in group 1 following the addition of DMQ to memantine were abdominal 
pain, diarrhoea, flatulence, nausea, decreased appetite, headache, dizziness, lethargy, somnolence, restlessness, asthenia and elevated alanine aminotransferase (ALT) [table III]. Clinically significant increased ALT values ranged from 50 to $156 \mathrm{U} / \mathrm{L}$ in group 1 . In group 2, the most common AEs following the addition of memantine to DMQ were dizziness, headache, dry mouth and elevated ALT values (table III). Clinically significant ALT values in group 2 ranged from 59 to $193 \mathrm{U} / \mathrm{L}$. AEs led to withdrawal of one subject in group 1, and three subjects in group 2. The subject who withdrew in group 1 had experienced nine central nervous system (CNS) and gastrointestinal AEs after 10 days of memantine monotherapy (i.e. 2 days after starting the $5 \mathrm{mg}$ every 12 hours dose); these AEs included lightheadedness, dizziness, insomnia, constipation, abdominal pain, nausea and loose stools, as well as emotional lability. Each of the three subjects who withdrew in group 2 also experienced multiple AEs, mostly involv- ing CNS and gastrointestinal problems, which all occurred from 1 to 3 days after the start of DMQ monotherapy.

No abnormalities in physical examination or vital signs were observed during the study. One haematology and several clinical chemistry values were out of range to a potentially clinical significant extent, mostly the hepatic enzymes ALT and aspartate aminotransferase (AST). The haematology result (elevated white blood cells) occurred at follow-up in one female who was told to consult with her family physician; the exact date of resolution is unknown. Three subjects in group 1 also had clinical chemistry values judged to be clinically significant, all documented on day 41 , including high ALT values in all three subjects $(50,56$ and up to $156 \mathrm{U} / \mathrm{L})$, and elevated AST in one subject $(72 \mathrm{U} / \mathrm{L})$; all values had returned to normal at follow-up. Three subjects in group 2 also had clinical chemistry values judged to be clinically significant. One subject, a 34-year-old

Table III. Summary of adverse event (AE) incidence, severity and type (occurring in at least two subjects during concomitant therapy)

\begin{tabular}{|c|c|c|c|c|}
\hline Variable & $\begin{array}{l}\text { Memantine monotherapy } \\
\text { (group 1, } \\
\text { days 22-32) } \\
{[n=20]}\end{array}$ & $\begin{array}{l}\text { DMQ + memantine } \\
\text { (group 1, } \\
\text { days } 33-40 \text { ) } \\
{[n=19]}\end{array}$ & $\begin{array}{l}\text { DMQ monotherapy } \\
\text { (group 2, } \\
\text { days 1-8) } \\
{[\mathrm{n}=28]}\end{array}$ & $\begin{array}{l}\text { Memantine + DMQ } \\
\text { (group 2, } \\
\text { days 30-40) } \\
{[n=20]}\end{array}$ \\
\hline Any $A E[n(\%)]$ & $9(45.0)$ & $14(73.7)$ & $25(89.3)$ & $14(70.0)$ \\
\hline \multicolumn{5}{|l|}{ AE severity [n (\%)] } \\
\hline Mild & $4(20.0)$ & $7(36.8)$ & $14(50.0)$ & $11(55.0)$ \\
\hline Moderate & $5(25.0)$ & $6(31.6)$ & $9(32.1)$ & $3(15.0)$ \\
\hline Severe & $0(0.0)$ & $1(5.3)$ & $2(7.1)$ & $0(0.0)$ \\
\hline \multicolumn{5}{|c|}{ AE (MedDRA preferred term) incidence [n (\%)] } \\
\hline Dizziness & $0(0.0)$ & $8(42.1)$ & $8(28.6)$ & $4(20.0)$ \\
\hline Diarrhoea & $0(0.0)$ & $7(36.8)$ & $6(21.4)$ & $1(5.0)$ \\
\hline Headache & $4(20.0)$ & $5(26.3)$ & $5(17.9)$ & $3(15.0)$ \\
\hline Nausea & $0(0.0)$ & $5(26.3)$ & $9(32.1)$ & $1(5.0)$ \\
\hline Somnolence & $3(13.0)$ & $3(15.8)$ & $8(28.6)$ & $0(0.0)$ \\
\hline Alanine aminotransferase increased & $0(0.0)$ & $3(15.8)$ & $0(0.0)$ & $2(10.0)$ \\
\hline Decreased appetite & $1(5.0)$ & $2(10.5)$ & $8(28.6)$ & $0(0.0)$ \\
\hline Lethargy & $0(0.0)$ & $2(10.5)$ & $0(0.0)$ & $0(0.0)$ \\
\hline Abdominal pain & $0(0.0)$ & $2(10.5)$ & $1(3.6)$ & $0(0.0)$ \\
\hline Asthenia & $0(0.0)$ & $2(10.5)$ & $3(10.7)$ & $0(0.0)$ \\
\hline Flatulence & $0(0.0)$ & $2(10.5)$ & $0(0.0)$ & $1(5.0)$ \\
\hline Restlessness & $0(0.0)$ & $2(10.5)$ & $0(0.0)$ & $0(0.0)$ \\
\hline Dry mouth & $2(10.0)$ & $0(0.0)$ & $3(10.7)$ & $2(10.0)$ \\
\hline
\end{tabular}

DMQ = dextromethorphan $30 \mathrm{mg}$ /quinidine $30 \mathrm{mg}$ every 12 hours; MedDRA = Medical Dictionary for Regulatory Activities. 
male, had high ALT (61 U/L) and gamma glutamyl transpeptidase (GGT; $89 \mathrm{U} / \mathrm{L}$ ) on day 28 , which remained elevated at follow-up (ALT $101 \mathrm{U} / \mathrm{L}$; GGT $200 \mathrm{U} / \mathrm{L}$ ), but had returned to normal, except for the GGT, at an unscheduled visit 2 months and 11 days after first dosing. The other two subjects in group 2 had elevated ALT values (up to 61 and $193 \mathrm{U} / \mathrm{L}$ ) on day 41, one of whom also had elevated AST (80 U/L), which had returned to normal at follow-up or at a later unscheduled visit. No relationship was observed between these chemistry value elevations and plasma concentrations of the drugs for the treatment groups, and almost all the values returned to normal.

\section{Secondary Outcomes: Pharmacodynamic Variables}

Few differences in pharmacodynamic parameters were observed among group 1 subjects on day 40 (DMQ + memantine) versus day 32 (memantine monotherapy), or in group 2 subjects on day 40 (memantine + DMQ) versus day 8 (DMQ monotherapy). The pharmacodynamic test results generally showed either minimal to no effect or a slight improvement with concomitant therapy, compared with monotherapy (see Supplemental Digital Content, http://links.adisonline.com/CDZ/ A5). The VAS for nausea showed no difference during concomitant therapy versus monotherapy; however, the VAS for dizziness showed a slight increase in dizziness at 2 and 4 hours post-dose with DMQ plus memantine therapy in group 1. For the BDI-II, BAI and LSEQ, in both groups the $90 \%$ CIs included 0 , indicating no differences in depression, anxiety or sleep scores, when DMQ was added to memantine or vice versa. Postural stability was slightly improved in group 1 when DMQ was added to memantine, but slightly decreased in several subtests (area of a 95\% confidence ellipsoid, eyes open and closed; average velocity of the centre-of-pressure coordinates, eyes open and closed) in group 2 when memantine was added to DMQ, compared with the respective monotherapies. Overall, no clinically meaningful adverse pharmacodynamic effects of the concomitant administration of DMQ and memantine were observed.

\section{Discussion}

This open-label study showed minimal or no drug interaction in healthy adult subjects resulting from coadministration of memantine and DMQ. This general lack of drug interaction was observed, regardless of the order in which the drugs were added, and in terms of pharmacokinetic, pharmacodynamic, and safety and tolerability measures, including AEs, laboratory values, vital signs and physical examination. The paucity of pharmacodynamic and $\mathrm{AE}$ findings on dual administration are especially noteworthy in light of concerns that dual NMDA blockade with memantine and DMQ could, theoretically, lead to increased risk of AEs, particularly of the CNS. ${ }^{[20]}$

The primary study outcomes $-90 \%$ CIs of the geometric mean ratios of monotherapy with memantine and DMQ versus combination therapy - were within the acceptance interval of $0.8-1.25$, as was $\mathrm{C}_{\text {max,ss, }}$ which would satisfy general requirements for bioequivalence testing. ${ }^{[33]}$ The $\mathrm{C}_{\min , \mathrm{ss}}$ of dextromethorphan was slightly higher when memantine was added to DMQ in group 2 subjects and the $90 \%$ CI of the geometric mean ratio slightly exceeded the upper limit of 1.25. In addition, slight increases in quinidine plasma concentrations $\left(\mathrm{AUC}_{12}, \mathrm{C}_{\mathrm{min}, \mathrm{ss}}\right.$ and $\mathrm{C}_{\text {max,ss }}$ ) were observed when memantine was added to DMQ in group 2 subjects, compared with DMQ monotherapy, which may be attributable to both quinidine and memantine undergoing renal secretion via similar renal cationic transport. ${ }^{[20,22-24]}$ However, as noted above, the low dose of quinidine in the DMQ $30 \mathrm{mg} / 30 \mathrm{mg}$ formulation resulted in total quinidine plasma concentrations that were near or below the LLQ $(0.05 \mu \mathrm{g} / \mathrm{mL})$, making it impossible to extract a $\log$ of zero; such data were given a value half of the LLQ, rendering these calculations unreliable. Moreover, the absolute mean difference in quinidine concentrations was small (about $0.03 \mu \mathrm{g} / \mathrm{mL}$ ), and therefore any effect of memantine on quinidine concentrations in DMQ is unlikely to be clinically relevant. With the exception of the above, no pharmacokinetic differences associated with concomitant memantine and DMQ compared with their respective monotherapies were 
observed. In addition, the DMQ dose used in this trial (dextromethorphan $30 \mathrm{mg} /$ quinidine $30 \mathrm{mg}$ ) was higher than the dose of the FDA-approved DMQ formulation (dextromethorphan $20 \mathrm{mg}$ / quinidine $10 \mathrm{mg}$ ); therefore, any potential for interaction between DMQ and memantine observed in a study of this design would likely be greater than any potential for interaction in clinical practice with concomitant use of these agents.

AEs were generally higher with DMQ monotherapy than with combination therapy. However, the overall incidence of AEs decreased with duration of DMQ treatment, suggesting that subjects were adjusting to the dose. As mentioned above, it is worth noting that the DMQ dosage used in this study was higher than the ultimately approved dosage for treatment of PBA; in addition, DMQ was not titrated as is recommended in the approved prescribing information document.

With regard to results for pharmacodynamic measures, no interaction was found with coadministration of DMQ and memantine on an array of tests covering neurological, cognitive, gastrointestinal and psychological parameters. Unexpectedly, performance on some subscales of the tests appeared to be improved with concomitant treatment, compared with either DMQ or memantine monotherapy. However, dizziness, as scored by VAS, was increased with the addition of DMQ to memantine in group 1 at 2 and 4 hours postdose. Several subtests of postural stability were also slightly decreased with the addition of memantine to DMQ in group 2; however, because none of the other postural stability measures were affected, this result did not appear to be clinically meaningful.

A limitation of this study is that it was open label; therefore, the pharmacodynamic results should be interpreted with caution. In addition, the study population was comprised of cognitively intact, healthy volunteers rather than elderly patients with dementia and PBA who would be likely recipients of combined memantine and DMQ therapy. Such older patients may also experience age-related changes in pharmacokinetic and pharmacodynamic factors that could result in unexpected drug effects. Nevertheless, the pharmacokinetic and pharmacodynamic results in this study in healthy volunteers suggest that there may be no meaningful drug interaction between DMQ and memantine.

\section{Conclusion}

Twice-daily coadministration of memantine $10 \mathrm{mg}$ and dextromethorphan $30 \mathrm{mg}$ /quinidine $30 \mathrm{mg}$ does not result in a clinically meaningful drug interaction, as reflected by the pharmacokinetic, pharmacodynamic or tolerability measures, regardless of whether DMQ is added to memantine at steady state or memantine is added to DMQ at steady state, although a small (20-30\%) increase in quinidine concentrations is possible. The results of this study suggest that memantine and DMQ could be safely coadministered without initial dose adjustment. However, as always when combining two drugs with overlapping pharmacology, patients should be monitored for potential increase in AEs.

\section{Acknowledgements}

Funding for this study was provided by Avanir Pharmaceuticals, Inc., which contributed to protocol development and manuscript preparation, but played no role in conducting the study or in data collection and analysis. Laura E. Pope is an employee of Avanir Pharmaceuticals, Inc. Kerri A. Schoedel and Cynthia Bartlett are employees of INC Research, which conducted the study. Edward M. Sellers was an employee of Ventana Clinical Research Corporation at the time the study was conducted. Editorial assistance in the form of substantive editing, copyediting and styling the manuscript was provided by The Curry Rockefeller Group, LLC, Tarrytown, NY, USA, and Andrea E. Formella, PharmD, Avanir Pharmaceuticals, Inc., and was funded by Avanir Pharmaceuticals, Inc.

\section{References}

1. Schiffer R, Pope LE. Review of pseudobulbar affect including a novel and potential therapy. J Neuropsychiatry Clin Neurosci 2005 Fall; 17 (4): 447-54

2. Wortzel HS, Oster TJ, Anderson CA, et al. Pathological laughing and crying: epidemiology, pathophysiology and treatment. CNS Drugs 2008; 22 (7): 531-45

3. Dark FL, McGrath JJ, Ron MA. Pathological laughing and crying. Aust N Z J Psychiatry 1996 Aug; 30 (4): 472-9

4. Davison C, Kelman H. Pathologic laughing and crying. Arch Neurol Psychiatry 1939 Oct; 42 (4): 595-643

5. Calvert T, Knapp P, House A. Psychological associations with emotionalism after stroke. J Neurol Neurosurg Psychiatry 1998 Dec; 65 (6): 928-9 
6. Tateno A, Jorge RE, Robinson RG. Pathological laughing and crying following traumatic brain injury. J Neuropsychiatry Clin Neurosci 2004 Fall; 16 (4): 426-34

7. Tang WK, Chen Y, Lam WW, et al. Emotional incontinence and executive function in ischemic stroke: a case-controlled study. J Int Neuropsychol Soc 2009 Jan; 15 (1): 62-8

8. Siddiqui MS, Fernandez HH, Garvan $\mathrm{CW}$, et al. Inappropriate crying and laughing in Parkinson disease and movement disorders. World J Biol Psychiatry 2009; 10 (3): 234-40

9. Petracca GM, Jorge RE, Ación L, et al. Frequency and correlates of involuntary emotional expression disorder in Parkinson's disease. J Neuropsych Clin Neurosci 2009 Fall; 21 (4): 406-12

10. Panitch HS, Thisted RA, Smith RA, et al., Pseudobulbar Affect in Multiple Sclerosis Study Group. Randomized, controlled trial of dextromethorphan/quinidine for pseudobulbar affect in multiple sclerosis. Ann Neurol 2006 May; 59 (5): 780-7

11. Brooks BR, Thisted RA, Appel SH, et al., AVP-923 ALS Study Group. Treatment of pseudobulbar affect in ALS with dextromethorphan/quinidine: a randomized trial. Neurology 2004 Oct; 63 (8): 1364-70

12. Pioro EP, Brooks BR, Cummings J, et al., Safety, Tolerability, and Efficacy Results Trial of AVP-923 in PBA Investigators. Dextromethorphan plus ultra low-dose quinidine reduces pseudobulbar affect. Ann Neurol 2010 Nov; 68 (5): 693-702

13. Nuedexta [package insert]. Aliso Viejo (CA): Avanir Pharmaceuticals, Inc; 2011

14. Choi DW, Peters S, Viseskul V. Dextrorphan and levorphanol selectively block N-methyl-D-aspartate receptormediated neurotoxicity on cortical neurons. J Pharmacol Exp Ther 1987 Aug; 242 (2): 713-20

15. Werling LL, Keller A, Frank JG, et al. A comparison of the binding profiles of dextromethorphan, memantine, fluoxetine and amitriptyline: treatment of involuntary emotional expression disorder. Exp Neurol 2007 Oct; 207 (2): 248-57

16. Musacchio JM, Klein M, Canoll PD. Dextromethorphan and sigma ligands: common sites but diverse effects. Life Sci 1989; 45 (19): 1721-32

17. Pope LE, Khalil MH, Berg JE, et al. Pharmacokinetics of dextromethorphan after single or multiple dosing in combination with quinidine in extensive and poor metabolizers. J Clin Pharmacol 2004 Oct; 44 (10): 1132-42

18. Walker FO, Hunt VP. An open label trial of dextromethorphan in Huntington's disease. Clin Neuropharmacol 1989 Aug; 12 (4): 322-30

19. Zhang Y, Britto MR, Valderhaug KL, et al. Dextromethorphan: enhancing its systemic availability by way of low-dose quinidine-mediated inhibition of cytochrome P4502D6. Clin Pharmacol Ther 1992 June; 51 (6): 647-55

20. European Medicines Agency. Ebixa ${ }^{\circledR}$ (memantine) $10 \mathrm{mg}$ film-coated tablets: summary of product characteristics [online]. Available from URL: http://www.ema.europa.eu/
docs/en_GB/document library/EPAR - Product Informa tion/human/000463/WC̄500058763.pdf [Accessed 2011 Oct 4]

21. Starkstein SE, Migliorelli R, Teson A, et al. Prevalence and clinical correlates of pathological affective display in Alzheimer's disease. J Neurol Neurosurg Psychiatry Jul 1995; 59 (1): $55-60$

22. Grace AA, Camm AJ. Quinidine. N Engl J Med 1998 Jan; 338 (1): $35-45$

23. Fattinger K, Vozeh S, Ha HR, et al. Population pharmacokinetics of quinidine. Br J Clin Pharmacol 1991 Mar; 31 (3): $279-86$

24. Rao N, Chou T, Ventura D, et al. Investigation of the pharmacokinetic and pharmacodynamic interactions between memantine and glyburide/metformin in healthy young subjects: a single-center, multiple-dose, open-label study. Clin Ther 2005 Oct; 27 (10): 1596-606

25. Ebixa ${ }^{\circledR}$ (memantine). Product monograph. Lundbeck. 2002 [online]. Available from URL: http://www.ebixa.com/con tent/downloadarea/pdf/monograph.pdf [Accessed 2011 Oct 4]

26. Stüven T, Griese EU, Kroemer HK, et al. Rapid detection of CYP2D6 null alleles by long distance- and multiplexpolymerase chain reaction. Pharmacogenetics 1996 Oct; 6 (5): 417-21

27. Hindmarch I, Shamsi Z, Stanley N, et al. A double-blind, placebo-controlled investigation of the effects of fexofenadine, loratadine and promethazine on cognitive and psychomotor function. Br J Clin Pharmacol 1999 Aug; 48 (2): $200-6$

28. Milovan D, Almeida L, Romach MK, et al. Effect of eslicarbazepine acetate and oxcarbazepine on cognition and psychomotor function in healthy volunteers. Epilepsy Behav 2010 Aug; 18 (4): 366-73

29. Collins JJ, De Luca CJ. Open-loop and closed-loop control of posture: a random-walk analysis of center-of-pressure trajectories. Exp Brain Res 1993; 95 (2): 308-18

30. Beck AT, Steer AT, Ball R, et al. Comparison of Beck Depression Inventories -1A and -II in psychiatric outpatients. J Pers Assess 1996 Dec; 67 (3): 588-97

31. Beck AT, Epstein N, Brown G, et al. An inventory for measuring clinical anxiety: psychometric properties. J Consult Clin Psychol 1988 Dec; 56 (6): 893-7

32. Parrott AS, Hindmarch I. The Leeds Sleep Evaluation Questionnaire in psychopharmacological investigations: a review. Psychopharmacology (Berl) 1980; 71 (2): 173-9

33. European Medicines Agency. Guideline on the investigation of bioequivalence. 2008 [online]. Available from URL: http://www.ema.europa.eu/docs/en_GB/document_library/ Scientific_guideline/2009/09/WC500003011.pdf [Accessed 2011 Jun 10]

Correspondence: Dr Laura E. Pope, PhD, Clinical Research, Avanir Pharmaceuticals, Inc., 20 Enterprise, Suite 200, Aliso Viejo, CA 92656, USA.

E-mail: Lpope@avanir.com 